\title{
Generalized Information Equilibrium Approaches to EEG Sleep Stage Discrimination
}

\author{
Todd Zorick ${ }^{1,2}$ and Jason Smith ${ }^{3}$ \\ ${ }^{1}$ Department of Psychiatry, Veterans Affairs Greater Los Angeles Healthcare System, Los Angeles, CA 90073, USA \\ ${ }^{2}$ Department of Psychiatry and Biobehavioral Sciences, UCLA, Los Angeles, CA, USA \\ ${ }^{3}$ The Boeing Company, Seattle, WA 98124, USA \\ Correspondence should be addressed to Todd Zorick; tzorick@mednet.ucla.edu
}

Received 15 March 2016; Revised 28 May 2016; Accepted 19 June 2016

Academic Editor: Valeri Makarov

Copyright (C) 2016 T. Zorick and J. Smith. This is an open access article distributed under the Creative Commons Attribution License, which permits unrestricted use, distribution, and reproduction in any medium, provided the original work is properly cited.

\begin{abstract}
Recent advances in neuroscience have raised the hypothesis that the underlying pattern of neuronal activation which results in electroencephalography (EEG) signals is via power-law distributed neuronal avalanches, while EEG signals are nonstationary. Therefore, spectral analysis of EEG may miss many properties inherent in such signals. A complete understanding of such dynamical systems requires knowledge of the underlying nonequilibrium thermodynamics. In recent work by Fielitz and Borchardt (2011, 2014), the concept of information equilibrium (IE) in information transfer processes has successfully characterized many different systems far from thermodynamic equilibrium. We utilized a publicly available database of polysomnogram EEG data from fourteen subjects with eight different one-minute tracings of sleep stage 2 and waking and an overlapping set of eleven subjects with eight different one-minute tracings of sleep stage 3. We applied principles of IE to model EEG as a system that transfers (equilibrates) information from the time domain to scalp-recorded voltages. We find that waking consciousness is readily distinguished from sleep stages 2 and 3 by several differences in mean information transfer constants. Principles of IE applied to EEG may therefore prove to be useful in the study of changes in brain function more generally.
\end{abstract}

\section{Introduction}

In electroencephalography (EEG), scalp electrodes measure electrical potential as a function of time [1]. EEG measures the sum of local field potentials in the region of cortex below the electrode, comprising $\sim 10^{9}$ cortical neurons [1]. EEG is typically analyzed by spectral analysis (Fourier transform) that assesses power in frequency bands [1]. However, many studies over the last 20 years have demonstrated that the underlying cortical neuronal dynamics is nonlinear and that EEG signals are nonstationary (the mean and variance change over time unpredictably). This has been mostly convincingly demonstrated both in vivo and in vitro using multielectrode arrays on cortical tissue, demonstrating the presence of "neuronal avalanches" $[2,3]$.

Given that the cortical neuronal dynamics largely responsible for the summed local field potentials that comprise EEG are characterized by scale-free avalanches consistent with a system at a critical state that is well described by power-law dynamics, many attempts have been made to analyze EEG using methods derived from fractal and other nonlinear theories, with some degree of success [49]. Another avenue of physical understanding of cortical avalanche dynamics would be via statistical physics and thermodynamics; however, the relatively large magnitude changes in scalp-recorded voltages in EEG clearly could not be characteristic of a system in thermodynamic equilibrium [10]. Therefore, a thorough statistical physics understanding of EEG would involve a complete description of cortical nonequilibrium thermodynamics, which is not possible for a noninvasive technique such as EEG [10, 11]. Similarly, previously published information-theoretic shortcuts to a thermodynamic understanding (such as maximum entropy approaches) for EEG suffer from insufficient knowledge of appropriate constraints for microscopic variables $[12,13]$. 
Instead, we propose to utilize the concept of generalized information transfer, where EEG could be modeled as an information transfer process [11, 14]. Generalized information equilibrium (IE) has been proposed as a systemindependent mechanism to study systems far from thermodynamic equilibrium, with applications to astrophysics, economics, materials science, Newtonian physics, and thermodynamics $[11,14,15]$. The principles of IE were developed from Hartley's original description [16] of an amount of information $(I)$ :

$$
I=K \cdot n
$$

where $n$ is the number of selected symbols and $K=\ln s$ is a constant which depends on the number of symbols $(s)$ available at each selection. Note that we use the natural logarithm, so that our natural information measure is in "nats" instead of "bits." Following Fielitz and Borchardt (2014) we will use the Hartley definition of information to say that the information in a given process $x$ is

$$
I_{x}=K_{x} \cdot n_{x} \quad \text { with } K_{x}=\ln s_{x},
$$

where $s_{x}$ is the size of the alphabet of symbols used to encode $x$ and $n_{x}$ is the number of symbols we select. A key assumption is that $n \gg 1$ (which we have from the $10^{8}$ to $10^{9}$ neurons in the cortex underlying an electrode).

Note that the more commonly utilized Shannon entropy (i) defined as [17]

$$
i=\frac{I}{n}=-\sum_{j=1}^{s} p_{j} \ln p_{j}
$$

reduces to the Hartley definition of information $(I)$ when the probability of each symbol in the alphabet is equal (i.e., $p_{j}$ is a constant). The use of Hartley's information theory, lacking any probabilistic assumptions, thus allows an estimation of information flow in any system even without access to knowledge of microscopic states or appropriate constraints in the case of maximum entropy approaches $[11,14]$. It should also be noted here that Hartley information is a special case of the Rényi entropy for $\alpha=0$ [18]:

$$
H_{\alpha}(X)=\frac{1}{1-\alpha} \ln \left(\sum_{i=1}^{n} p_{i}^{\alpha}\right) .
$$

It has been demonstrated that one can use Hartley's information theory to define a natural amount of information for any system $[11,14]$ :

$$
I_{x}=\kappa_{x} \frac{|\Delta x|}{|\delta x|}
$$

where $\kappa_{x}$ is the information transfer constant, $|\Delta x|$ is the absolute value, and $|\delta x|$ is the signal of the process variable $x$, with $|\delta x| \ll|\Delta x|$. Using this relationship, virtually any system where information flows from a source $(y)$ to a destination $(x)$ can be considered from the point of view of information transfer [11, 14]. The important point is, however, that the amount of information (I) must generally obey the inequality

$$
I_{x} \leq I_{y}
$$

when the process variable $x$ is related to the information destination and the process variable $y$ to the information source. For the current study, we assume ideal information transfer $\left(I_{x}=I_{y}\right)$ and, hence, information equilibrium (IE). Considering (5) one gets

$$
\left(\frac{|\delta y|}{|\delta x|}\right)=\frac{K_{y}}{K_{x}} \frac{|\Delta y|}{|\Delta x|} .
$$

For convenience we will denote the ratio $\kappa_{y} / \kappa_{x}$ as $\kappa$ and call it the information transfer constant for ideal information transfer or for IE. For EEG, we use (7) to define an information transfer constant $(\kappa)$ for each time interval $(\Delta t)$ to the voltage reading $(|\Delta V|)$. We analyze the distribution of $\kappa$ values to see if they are peaked around a well-defined mean. In that case we can interpret (7) (for small changes in the process variables $d x$ and $d y$ ) as a differential equation:

$$
\frac{d|\Delta V|}{d|\Delta t|}=\kappa_{\mathrm{avg}} \frac{|\Delta V|}{|\Delta t|},
$$

which has the solution

$$
|\Delta V| \sim|\Delta t|^{\kappa_{\text {avg }}} .
$$

We will make a few observations here about the IE approach and its relationship to other physical descriptions of dynamic systems. For general information equilibrium, the solution to (8) can be rewritten as

$$
|\Delta V|_{t_{0}+\Delta t}=|\Delta V|_{t_{0}} \exp \left(\kappa \log \left(\frac{t_{0}+\Delta t}{t_{0}}\right)\right) .
$$

Let us now set a new parameter, $\lambda=\kappa t_{0}$. Over short time scales $\left(t_{0} \gg \Delta t\right),(10)$ reduces to

$$
|\Delta V|_{t_{0}+\Delta t}=|\Delta V|_{t_{0}} \exp \lambda \Delta t .
$$

Equation (11) is precisely the form of a Lyapunov exponent if the voltage measurement is considered as a superposition of a large number of neurons at different distances from the EEG sensor (i.e., $|\Delta V|$ is a sum over $n$ individual neuron voltages near the sensor, mapping a $4 n$ dimensional "phase space" to a voltage measurement $\left.\left(\mathbb{R}^{3} \times V\right)^{n} \rightarrow|V|\right)$. Lyapunov exponents are deeply related to the study of chaotic dynamical systems, with positive values indicating a chaotic system with exponential divergence from initial conditions [19]. For systems with power-law sensitivity to initial conditions, Lyapunov exponent analysis has been generalized to the scale-dependent Lyapunov exponent, which has been utilized to successfully describe many dynamic physical systems, including EEG-based seizure identification in humans (e.g., $[5,20-22])$.

For the current study, we utilize a publicly available database of polysomnographic data for fourteen subjects with eight minutes each of waking and sleep stage 2 EEG (and eleven subjects with eight minutes of sleep stage 3 EEG) to assess for differences in patterns of $\kappa$ values to assess the utility of IE in distinguishing different states of consciousness. Our hypothesis is that different states of consciousness can be identified by different distributions of $\kappa$ and different $\kappa_{\text {avg }}$ values. 
TABLE 1: Mean information transfer ratio comparisons.

\begin{tabular}{|c|c|c|c|c|c|}
\hline$\Delta T$ & Waking versus & Sleep stage 2 & $F_{(1,221)}$ & Sleep stage 3 & $F_{(1,184)}$ \\
\hline 0.004 & $1.08(0.097)$ & $1.21(0.08)$ & $170.8^{* * *}$ & $1.17(0.08)$ & $144.1^{* * *}$ \\
\hline 0.04 & $1.60(0.18)$ & $1.65(0.12)$ & $19.8^{* * *}$ & $1.57(0.14)$ & 1.67 \\
\hline 0.4 & $5.51(0.48)$ & $5.31(0.47)$ & $19.1^{* * *}$ & $4.51(0.57)$ & $263.1^{* * *}$ \\
\hline 4 & $-3.62(0.33)$ & $-3.53(0.31)$ & $9.6^{* *}$ & $-3.01(0.36)$ & $280.1^{* * *}$ \\
\hline
\end{tabular}

$\Delta T$ : time difference for voltage change calculations, in seconds.

Values represent mean (s.d.) for group mean information transfer ratio $(\kappa)$.

${ }^{* * *} p<0.0001 ;{ }^{* *} p<0.001$ after Bonferroni correction.

\section{Materials and Methods}

2.1. Database. We utilized a publicly available EEG dataset (slpdb) http://www.physionet.org/, which was a polysomnogram study of patients with severe sleep apnea [23]. There were $n=14$ subjects with 8 min of waking EEG and sleep stage 2 EEG and $n=11$ subjects with $8 \mathrm{~min}$ of sleep stage 3 EEG. An additional dataset of $n=13$ subjects of waking EEG, $n=10$ subjects of REM sleep EEG, and $n=8$ subjects of sleep stage 1 EEG (1 minute each, nonoverlapping with the larger 8 min EEG dataset) was also generated from the larger dataset. The exact dataset used has previously been described in a prior unrelated study [9]. EEG segments chosen for further analysis were selected on the basis of the absence of movement artifacts and disordered breathing, which limited the amount of suitable tracings. No demographic and limited clinical information was available from the dataset. Digitized $250 \mathrm{~Hz}$ EEG recordings on a 10-20 international system were used with a single EEG lead for each subject, which differed among subjects; no information was provided about reference electrode placement [9]. Use of the dataset for this study was approved by the VA West Los Angeles IRB.

2.2. $\kappa$ Estimation. EEG is a time series of voltage readings $V(t)$, where $t=1,2, \ldots, n$ (length of series) for each value of $t$ up to $n-\Delta t$, given a time interval $\Delta t$, so the $\kappa$ values for each instant can be calculated:

$$
\kappa_{\Delta t}=\frac{\log \left(\left|V_{t+\Delta t}-V_{t}\right|\right)}{\log (|\Delta t|)} .
$$

Therefore, each segment of EEG would be characterized by a series of information transfer constant ratios, for different values of the time interval $\Delta t$ (i.e., 1, 2, 4, 8, time steps, etc.), and for each segment the mean $\kappa_{\text {avg }}$ was calculated:

$$
\kappa_{\mathrm{avg}}=\frac{1}{n-\Delta t} \sum_{t=1}^{n-\Delta t} \kappa_{\Delta t} .
$$

Code for extracting $\kappa$ values from EEG was written in $\mathrm{R}$ [24]. We used the natural log for transformation throughout. Values where $V(t)=V(t+\Delta t)$ were excluded from estimation (as the logarithm of zero is undefined).

2.3. Analyses. Probability density function (PDF) estimation was done using the $\mathrm{R}$ density package. Lomb-Scargle periodograms were done using the $\mathrm{R}$ package cts [25], designed to follow [26]. To assess statistically significant periodogram peaks, we utilized a $p \leq 0.01$ threshold, heuristically estimating the maximum possible number of frequencies in the input PDF as twice the number of data points in the PDF [26]. All statistics were done in R [24]. For the REM sleep and sleep stage 1 dataset analysis with the reduced size dataset, we utilized generalized linear mixed modeling (GLMM) with unstructured covariance matrices to account for subject-specific effects, using the R package nlme [27].

\section{Results}

3.1. Waking Differs from Sleep Stages 2 and 3 in $\kappa$ Values at Multiple Time Scales. We calculated the mean $\kappa$ value for each segment in our database with a range of different $\Delta T$ values (0.004, 0.04, 0.4, and 4 seconds; Figure 1, Table 1). An example of the comparison in the PDFs of $\kappa$ values for all three states of consciousness for $1 \mathrm{~min}$ each of EEG for a single subject at $\Delta T$ values from 0.004 to $4 \mathrm{sec}$ is shown in Figure 1 .

Segment-specific mean $\kappa$ values were then analyzed by repeated-measures ANOVA with state of consciousness as the grouping variable and subject as the repeated measure (Table 1). These results demonstrate that waking EEG is clearly distinguishable from sleep stages 2 and 3 via segment mean $\kappa$ values (Table 1 ). While waking and sleep stage 3 $\kappa$ values differ strongly at $0.004-, 0.4-$, and 4 -second time scales, there is no difference between them at the 0.04-second time scale (Table 1). Interestingly, while there seems to be a pattern for waking and sleep stage $2 \kappa$ values to slowly become less different over time, if anything the opposite is true for the waking/sleep stage 3 comparison, where the 4second time scale shows the largest difference between the two (comparing $F$ values; Table 1 ).

3.2. Greater Proportion of Low Information Transfer at $\Delta T$ of $0.004 \mathrm{sec}$ in Sleep Stages 2 and 3. Next, we assessed $\kappa$ values for the proportion in each segment with values $<0.2$, as a heuristic indicator of low information transfer at the $250 \mathrm{~Hz}$ sampling rate (Table 2). Strikingly, both sleep stage 2 and (more so) sleep stage 3 have a greater proportion of low information transfer $\kappa$ values than waking EEG (Table 2). At larger time steps, however, there was no difference between consciousness stages in the proportion of low information transfer (data not shown).

3.3. Waking Differs from Sleep Stages 2 and 3 in the Extent of Periodicity in the PDFs of $\kappa$ Values at All Time Scales. As can be noted in Figure 1, there appears to be a periodicity in the 


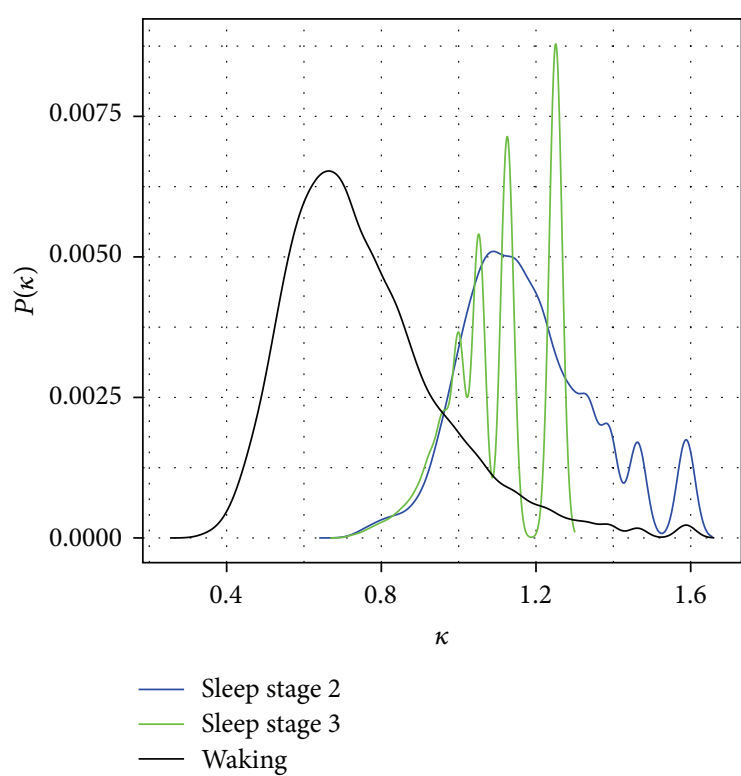

(a)

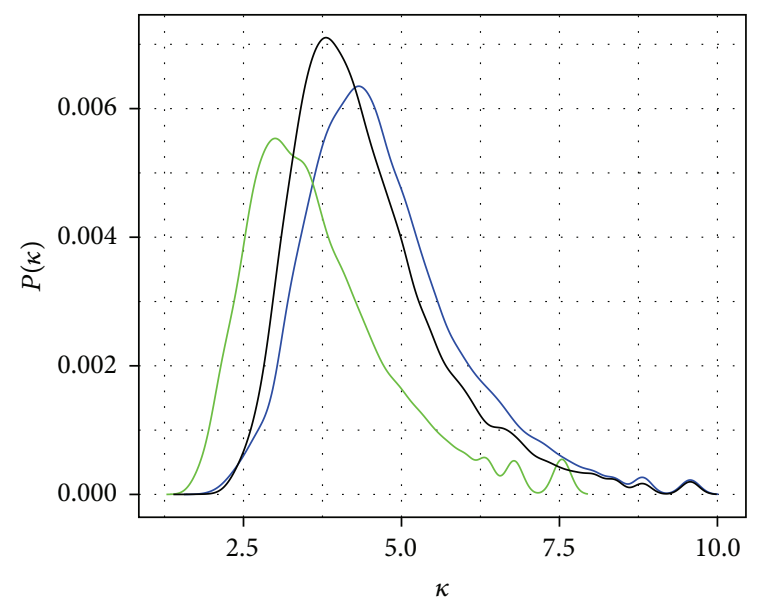

- Sleep stage 2
_ Sleep stage 3
- Waking

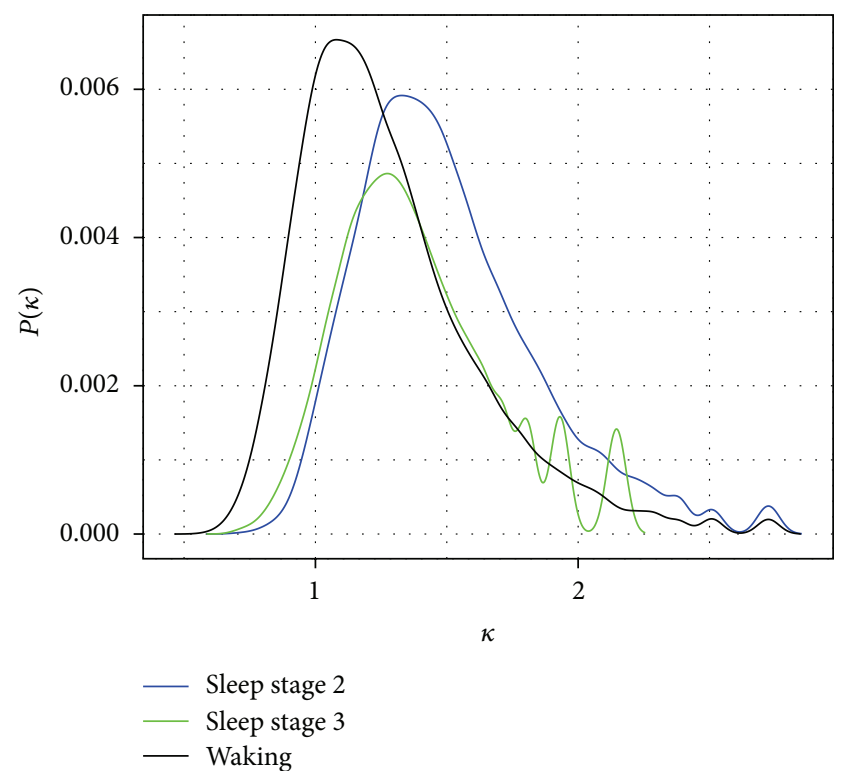

(b)

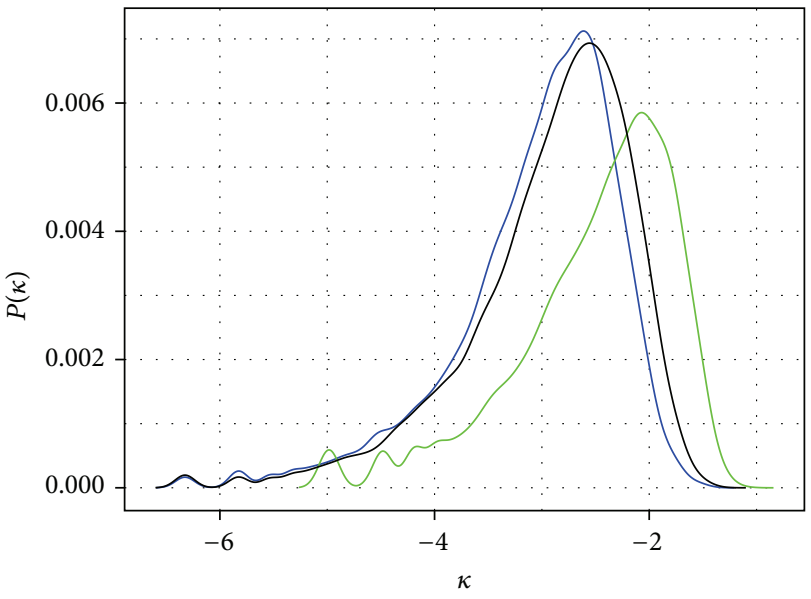

Sleep stage 2
_ Sleep stage 3
W Waking

(c)

(d)

FIGURE 1: PDF estimations of $\kappa$ values for one subject. One minute each of EEG from waking (black), sleep stage 2 (blue), and sleep stage 3 (green) from a single subject was analyzed for $\kappa$ values and the PDF estimated and plotted for each of (a) $\Delta T=0.004 \mathrm{sec}$; (b) $\Delta T=0.04 \mathrm{sec}$; (c) $\Delta T=0.4 \mathrm{sec}$; (d) $\Delta T=4 \mathrm{sec} . P(\kappa)$ : frequency of $\kappa$ values. Note the characteristic amplitude fluctuations at all scales for the largest magnitude $\kappa$ values.

TABLE 2: Fraction of values with kappa $<0.2$ at $\Delta T=0.004 \mathrm{~s}$.

\begin{tabular}{lcc}
\hline Waking versus & Sleep stage 2 & $F_{(1,209)}$ \\
\hline $0.018(0.020)$ & $0.031(0.023)$ & $\mathbf{7 5 3 . 2 ^ { * * * }}$ \\
\hline $0.018(0.020)$ & Sleep stage 3 & $F_{(1,184)}$ \\
\hline
\end{tabular}

Data represent mean (s.d.) for fraction of $\kappa$ values $<0.2$.

Per segment, via repeated-measures ANOVA. ${ }^{* * *} p<0.0001$

$\mathrm{PDF}$ of $\kappa$ values, in that certain magnitudes are enhanced, and others are diminished. In order to quantify this, we made PDF estimations for $\kappa$ values for all segments and then performed a normalized Lomb-Scargle periodogram analysis, in order to assess for periodicity (Figure 2; Table 3). Sleep stage 2 exhibits enhanced periodicity, while sleep stage 3 shows diminished periodicity compared with waking at the $0.004 \mathrm{sec}$ time step. For all other time steps, though, sleep stage 3 shows greater periodicity in the $\kappa$ value PDF estimations than sleep stage 2 and waking consciousness (Figure 2; Table 3). Note that although sleep stage 2 and waking appear to be very similar at all other time steps (Figure 2), there are in fact modest quantitative differences between their periodicities (Table 3). 
TABle 3: Mean $p \geq 0.01$ Lomb-Scargle power comparisons.

\begin{tabular}{lcccc}
\hline$\Delta T(\mathrm{sec})$ & Waking & Sleep stage 2 & $F_{(1,209)}$ & Sleep stage 3 \\
\hline $\mathbf{0 . 0 0 4}$ & $1.2 \times 10^{5}\left(2.2 \times 10^{4}\right)$ & $1.5 \times 10^{5}\left(2.7 \times 10^{4}\right)$ & $F_{(1,184)}$ \\
$\mathbf{0 . 0 4}$ & $9.8 \times 10^{4}\left(2.2 \times 10^{4}\right)$ & $1.0 \times 10^{5}\left(2.2 \times 10^{4}\right)$ & $\mathbf{1 1 . 8 7 ^ { * * }}$ & $9.1 \times 10^{4}\left(5.8 \times 10^{4}\right)$ \\
$\mathbf{0 . 4}$ & $3.5 \times 10^{4}\left(6.7 \times 10^{3}\right)$ & $3.3 \times 10^{4}\left(5.1 \times 10^{3}\right)$ & $\mathbf{6 2 . 6 ^ { * * * }}$ \\
$\mathbf{4}$ & $5.2 \times 10^{4}\left(9.5 \times 10^{3}\right)$ & $5.0 \times 10^{4}\left(7.6 \times 10^{3}\right)$ & $\mathbf{1 1 . 0 8 ^ { * * }}$ & $4.8 \times 10^{4}\left(1.6 \times 10^{4}\right)$ \\
\hline
\end{tabular}

Listed values represent mean (s.d.) of $p \leq 0.01$ Lomb-Scargle periodogram power for each state of consciousness. Comparisons were done by repeatedmeasures ANOVA. ${ }^{* * *} p<0.0001 ;{ }^{* *} p<0.01$.

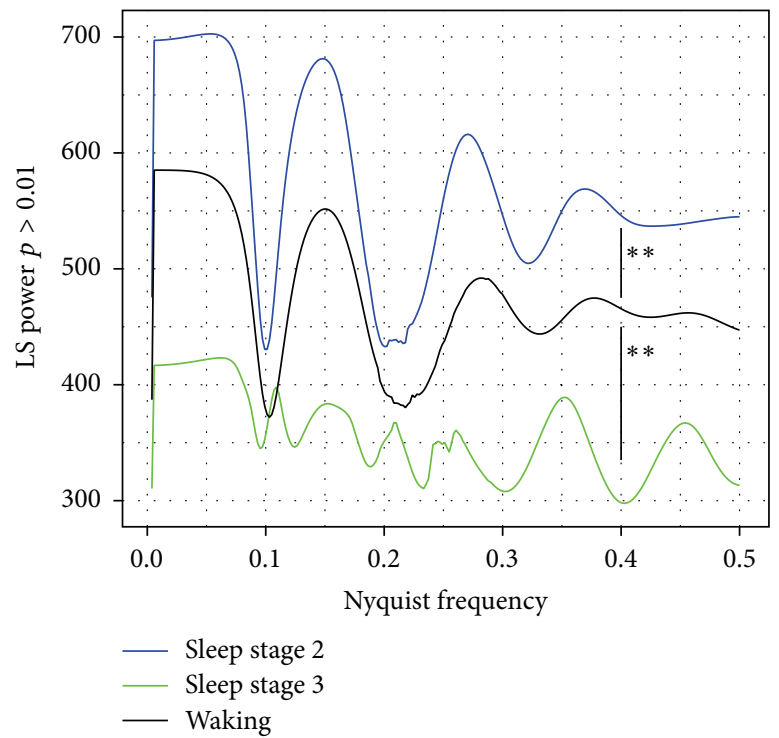

(a)

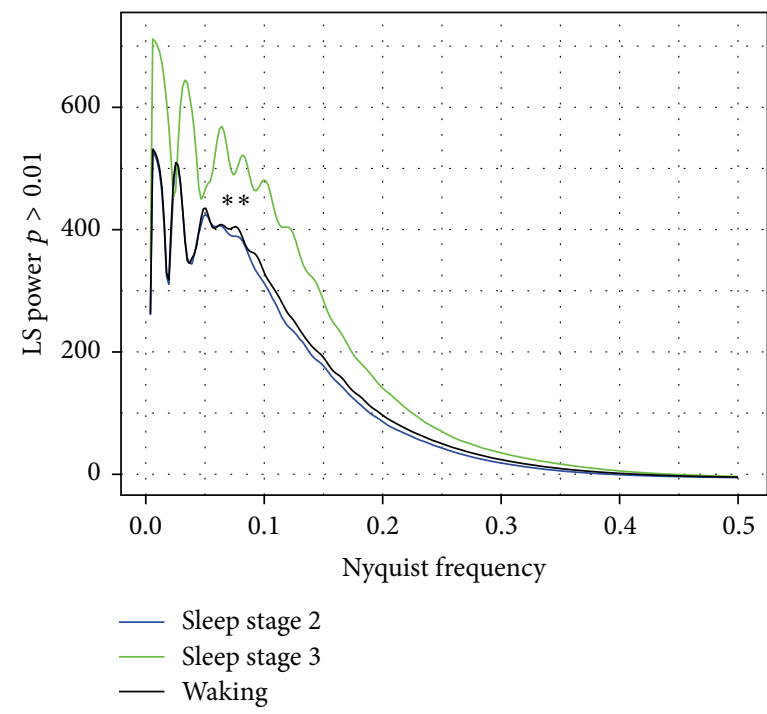

(c)

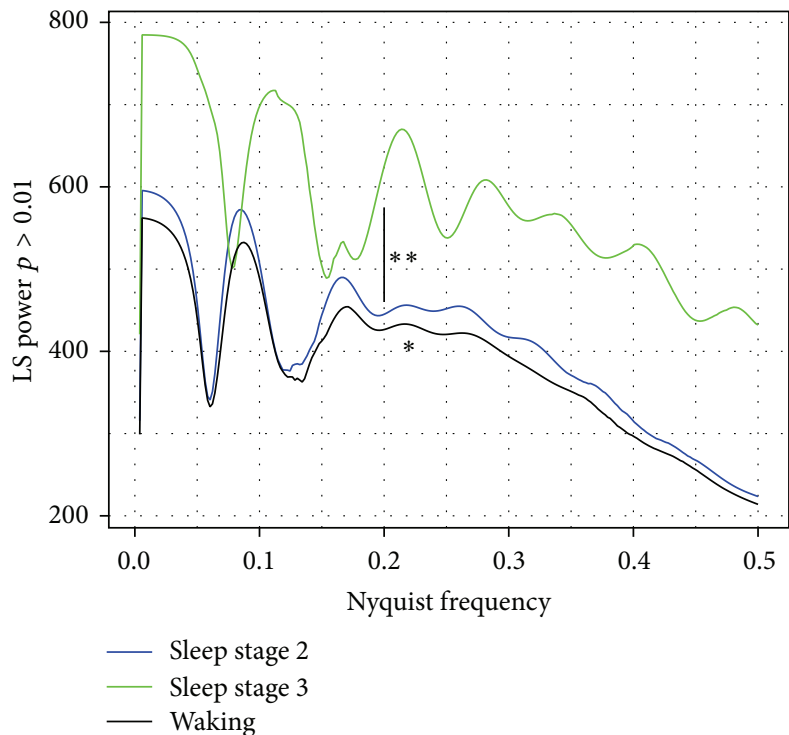

(b)

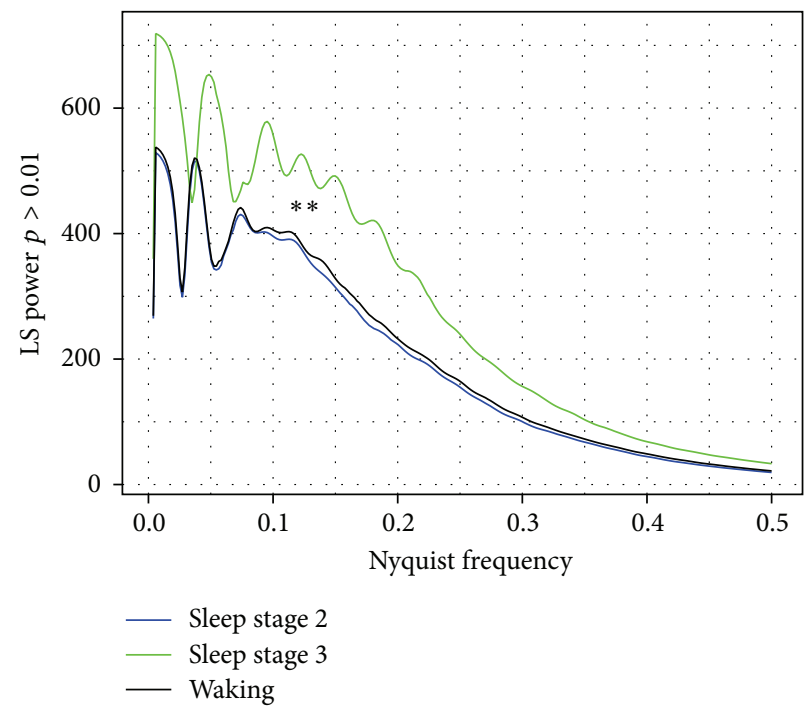

(d)

FIGURE 2: Lomb-Scargle periodogram power for $\kappa$ value PDF estimations. Lines represent mean data for EEG of $n=14$ subjects from waking (black) and sleep stage 2 (blue) and $n=11$ subjects from sleep stage 3 (green), each with eight different one-minute segments. Data was analyzed for $\kappa$ values and the PDF estimated and then normalized Lomb-Scargle periodogram area with a threshold of $p>0.01$ calculated. (a) $\Delta T=0.004 \mathrm{sec}$; (b) $\Delta T=0.04 \mathrm{sec}$; (c) $\Delta T=0.4 \mathrm{sec}$; (d) $\Delta T=4 \mathrm{sec}$. ${ }^{* *} p<0.0001$ by repeated-measures ANOVA for Lomb-Scargle periodogram area difference between waking and sleep stage 2 (a) and waking and sleep stage 3 (a-d); ${ }^{*} p<0.01$ between waking and sleep stage 2 (b). 
TABLE 4: Mean $\kappa$ and LS power for sleep stage 1 and REM sleep versus waking EEG.

\begin{tabular}{|c|c|c|c|c|c|}
\hline$\Delta T(\mathrm{sec})$ & Waking & Sleep stage 1 & $t$ stat & REM sleep & $t$ stat \\
\hline \multicolumn{6}{|c|}{ Mean $\kappa^{\mathrm{a}}$} \\
\hline 0.004 & $1.06(0.09)$ & $1.17(0.1)$ & $2.46^{*}$ & $1.27(0.09)$ & $5.61^{* * *}$ \\
\hline 0.04 & $1.56(0.19)$ & $1.69(0.08)$ & 2.04 & $1.75(0.16)$ & $4.15^{* *}$ \\
\hline 0.4 & $5.39(0.54)$ & $5.69(0.42)$ & 2.42 & $5.74(0.59)$ & 2.31 \\
\hline 4 & $-3.45(0.37)$ & $-3.62(0.23)$ & 1.4 & $-3.66(0.41)$ & 1.54 \\
\hline \multicolumn{6}{|c|}{ LS power ${ }^{\mathrm{b}}$} \\
\hline 0.004 & $7879(4989)$ & $7766(4826)$ & 0.05 & $8422(9083)$ & 0.18 \\
\hline 0.04 & $1.7 \times 10^{5}\left(5.2 \times 10^{4}\right)$ & $1.9 \times 10^{5}\left(3.7 \times 10^{4}\right)$ & 0.93 & $2.1 \times 10^{5}\left(6.2 \times 10^{4}\right)$ & $3.26^{*}$ \\
\hline 0.4 & $2.9 \times 10^{5}\left(8.3 \times 10^{4}\right)$ & $3.7 \times 10^{5}\left(9.4 \times 10^{4}\right)$ & 2.12 & $4.0 \times 10^{5}\left(1.3 \times 10^{5}\right)$ & $3.32^{*}$ \\
\hline 4 & $4.5 \times 10^{5}\left(1.3 \times 10^{5}\right)$ & $6.2 \times 10^{5}\left(1.9 \times 10^{5}\right)$ & $2.63^{*}$ & $6.3 \times 10^{5}\left(2.0 \times 10^{5}\right)$ & $2.76^{*}$ \\
\hline
\end{tabular}

${ }^{a}$ Listed values represent mean (s.d.) of $\kappa$ values for each state.

${ }^{\mathrm{b}}$ Listed values represent mean (s.d.) of $p \leq 0.01$ Lomb-Scargle periodogram power for each state.

Comparisons were done by GLMM. ${ }^{* * *} p<0.001 ;{ }^{* *} p<0.01 ;{ }^{*} p<0.05$; bold: $p<0.1$.

3.4. Waking Also Differs from Sleep Stage 1 and REM Sleep in Both Mean $\kappa$ Values and the Extent of Periodicity in the PDFs of $\kappa$ Values at Various Time Scales. Given the limitations of the physionet.org dataset, only a limited number of segments with artifact-free sleep stage 1 and REM sleep EEGs were available. As a further test of the IE description of EEG, we compared a nonoverlapping group of 1-minute tracings of waking, sleep stage 1 , and REM sleep EEG by mean $\kappa$ and Lomb-Scargle periodogram analysis of $\kappa$ PDFs, using generalized linear mixed modeling (GLMM; Table 4). Neither sleep stage 1 nor REM sleep exhibited a significant proportion of low information transfer $\kappa$ values at the sampling rate (data not shown). In terms of mean $\kappa$ values, sleep stage 1 exhibits a larger value than waking only at the $250 \mathrm{~Hz}$ sampling rate, with only a trend towards significance at $\Delta t=0.04$ and $0.4 \mathrm{sec}$, and no difference at $\Delta t=4 \mathrm{sec}$ (Table 4 ). REM sleep mean $\kappa$ values are larger than those for waking EEG at the sampling rate and $\Delta t=0.04 \mathrm{sec}$, with only a trend at $\Delta t=0.4 \mathrm{sec}$, with no difference between states at $\Delta t=4 \mathrm{sec}$ (Table 4 ).

Interestingly, both sleep stage 1 and REM sleep exhibited no difference from waking EEG in terms of the extent of periodicity in the $\kappa$ value PDF estimations at the sampling rate, whereas both showed stronger differences with larger time steps (Table 4). Sleep stage 1 did not differ from waking in periodicity in the $\kappa$ value PDF estimations at $\Delta t=0.04 \mathrm{sec}$, demonstrated a trend towards an increase in periodicity at $\Delta t$ $=0.4 \mathrm{sec}$, and showed an increase in periodicity at $\Delta t=4 \mathrm{sec}$ (Table 4). By contrast, REM sleep exhibited a greater degree of periodicity in the $\kappa$ value PDF estimations than waking at $\Delta t=0.04,0.4$, and $4 \mathrm{sec}$.

\section{Discussion}

4.1. Generalized Information Theory and EEG. To our knowledge, this report is the first application of principles of IE to the study of EEG. Other information-theoretic approaches have a long history of neuroscience applications [28], but maximum entropy applications to EEG in particular have been limited by an appropriate understanding of microscopic constraints $[12,13]$. The theoretical advantage of IE approaches to the analysis of EEG (or any other system) is that an explicit probabilistic understanding of the underlying system states (i.e., cortical local field potentials) is unnecessary; thus an estimation of "natural" information transfer can be assessed from a macro-observable (like time-dependent scalp voltage) alone [11, 14].

4.2. Utility of IE for the Study of Sleep Stage Discrimination. Our study demonstrates several interesting findings with regard to the application of IE to the analysis of polysomnogram data in EEG. Firstly, there is a clear distinction between waking, sleep stage 2 , and sleep stage 3 consciousness in terms of mean $\kappa$ values across different time scales (Table 1 ), with sleep stage 1 and REM sleep consciousness having distinction at fewer time scales (Table 4). Secondly, waking differs from both sleep stage 2 and sleep stage 3 (but not sleep stage 1 or REM sleep) in terms of the proportion of low information transfer $\kappa$ values at the sampling rate $(250 \mathrm{~Hz})$. Thirdly and perhaps most surprisingly, there is a clear periodicity of the PDF of the $\kappa$ values at all time scales, which differs strongly between waking, sleep stage 2 , and sleep stage 3 , with more limited differences between waking and sleep stage 1 and REM sleep (Figure 2, Tables 3 and 4). Thus, there is an overall richness of the IE description of differences in sleep and consciousness states that may well suit it to be used as a general tool to study states of altered cortical function. Indeed, the apparent discriminative power of IE (Tables 14) for sleep staging compares favorably with many other descriptions of computer-based analytic techniques for EEG, including fractals [6], multifractals [7, 9, 29, 30], and Tsallis entropy [4], not to mention automatic feature extraction from spectral analysis (reviewed in [31]).

4.3. Limitations. We utilized a publicly available dataset with minimal clinical or demographic information available. The number of subjects available was relatively small, and the number of EEG segments available was smaller still for sleep stage 1 and REM sleep. Waking consciousness is likely to be a heterogeneous state of brain activities; thus identifying this state only based upon clinical polysomnogram staging may limit the ability of any technique to assess for differences between waking and sleep stages. We can not exclude 
the possibility that some of the observed differences between states of consciousness were caused by differences in motor or muscle activity.

\section{Conclusions}

Given the highly significant results from application of IE to EEG, with the ability to discriminate between waking and sleep consciousness stages via multiple distinct statistical descriptions, the study of EEG-based information transfer constant $(\kappa)$ certainly deserves to be tried more generally with other sleep EEG datasets to ensure replicability. The application of IE to EEG is very straightforward, with extremely simple programming algorithms compared to other techniques. Indeed, if the results of the present study are a guide, it may be interesting to apply IE more widely with states of brain dysfunction to see if it will become a useful tool in the quantitative analysis of EEG.

\section{Abbreviations}

IE: Information equilibrium.

\section{Competing Interests}

The authors declare that there are no competing interests.

\section{Acknowledgments}

Todd Zorick is supported by a United States Veterans Administration Career Development Award no. I01 BX007080. The authors thank Drs. P. Fielitz and G. Borchardt for helpful discussions and comments on the paper.

\section{References}

[1] P. L. Nunez and R. Srinivasan, Electric Fields of the Brain: The Neurophysics of EEG, Oxford University Press, New York, NY, USA, 2nd edition, 2006.

[2] A. Klaus, S. Yu, and D. Plenz, "Statistical analyses support power law distributions found in neuronal avalanches," PLOS ONE, vol. 6, no. 5, article e19779, 2011.

[3] D. Plenz and T. C. Thiagarajan, "The organizing principles of neuronal avalanches: cell assemblies in the cortex?" Trends in Neurosciences, vol. 30, no. 3, pp. 101-110, 2007.

[4] S. M. Cai, Z. H. Jiang, T. Zhou, P. L. Zhou, H. J. Yang, and B. H. Wang, "Scale invariance of human electroencephalogram signals in sleep," Physical Review E: Statistical, Nonlinear, and Soft Matter Physics, vol. 76, no. 6, part 1, Article ID 061903, 2007.

[5] J. Gao, J. Hu, and W.-W. Tung, "Complexity measures of brain wave dynamics," Cognitive Neurodynamics, vol. 5, no. 2, pp. 171182, 2011.

[6] J.-M. Lee, D.-J. Kim, I.-Y. Kim, K.-S. Park, and S. I. Kim, "Detrended fluctuation analysis of EEG in sleep apnea using MIT/BIH polysomnography data," Computers in Biology and Medicine, vol. 32, no. 1, pp. 37-47, 2002.

[7] M. Qianli, N. Xinbao, W. Jun, and L. Jing, "Sleep-stage characterization by nonlinear EEG analysis using wavelet-based multifractal formalism," in Proceedings of the in 27th Engineering in
Medicine and Biology Society (IEEE-EMBS '05), pp. 4526-4529, Shanghai, China, 2005.

[8] C. J. Stam, "Nonlinear dynamical analysis of EEG and MEG: review of an emerging field," Clinical Neurophysiology, vol. 116, no. 10, pp. 2266-2301, 2005.

[9] T. Zorick and M. A. Mandelkern, "Multifractal detrended fluctuation analysis of human EEG: preliminary investigation and comparison with the wavelet transform modulus maxima technique," PLoS ONE, vol. 8, no. 7, Article ID e68360, 2013.

[10] T. L. Hill, An Introduction to Statistical Thermodynamics, Dover Publications, New York, NY, USA, 2nd edition, 1986.

[11] P. Fielitz and G. Borchardt, "A general concept of natural information equilibrium: from the ideal gas law to the $\mathrm{K}$ Trumpler effect," 2014, https://arxiv.org/abs/0905.0610.

[12] W. Boomsma, J. Ferkinghoff-Borg, and K. Lindorff-Larsen, "Combining experiments and simulations using the maximum entropy principle," PLoS Computational Biology, vol. 10, no. 2, article e1003406, 2014.

[13] W. Truccolo, O. J. Ahmed, M. T. Harrison et al., "Neuronal ensemble synchrony during human focal seizures," The Journal of Neuroscience, vol. 34, no. 30, pp. 9927-9944, 2014.

[14] P. Fielitz and G. Borchardt, "A generalized concept of information transfer," Physics Essays, vol. 24, no. 3, pp. 350-363, 2011.

[15] J. Smith, "Information equilibrium as an economic principle," http://arxiv.org/abs/1510.02435.

[16] R. V. L. Hartley, “Transmission of Information,” The Bell System Technical Journal, vol. 7, no. 3, pp. 535-563, 1928.

[17] C. E. Shannon, "A mathematical theory of communication," The Bell System Technical Journal, vol. 27, pp. 379-423, 1948.

[18] N. L. Bhamidipati, "Renyi Entropy," Mathworld-A Wolfram Web Resource, Created by Eric W. Weisstein, 2016.

[19] E. W. Weisstein, Lyapunov Characteristic Exponent, Wolfram MathWorld Web Resource, 2016.

[20] J. Gao, Y. Cao, W. W. Tung, and J. Hu, Multiscale Analysis of Complex Time Series: Integration of Chaos and Random Fractal Theory, and Beyond, Wiley-Interscience, Hoboken, NJ, USA, 2007.

[21] J. Gao and J. Hu, "Fast monitoring of epileptic seizures using recurrence time statistics of electroencephalography," Frontiers in Computational Neuroscience, vol. 7, article 122, 2013.

[22] J. B. Gao, J. Hu, W. W. Tung, and Y. H. Cao, "Distinguishing chaos from noise by scale-dependent Lyapunov exponent," Physical Review E: Statistical, Nonlinear, and Soft Matter Physics, vol. 74, no. 6, part 2, Article ID 066204, 2006.

[23] Y. Ichimaru and G. B. Moody, "Development of the polysomnographic database on CD-ROM," Psychiatry and Clinical Neurosciences, vol. 53, no. 2, pp. 175-177, 1999.

[24] RCoreTeam, R: A Language and Environment for Statistical Computing, R Foundation for Statistical Computing, Vienna, Austria, 2015.

[25] Z. Wang, "cts: an $R$ package for continuous time autoregressive models via Kalman filter," Journal of Statistical Software, vol. 53, no. 5, pp. 1-19, 2013.

[26] W. H. Press, S. A. Teukolsky, W. T. Vetterling, and B. P. Flannery, Numerical Recipes: The Art of Scientific Computing, Cambridge University Press, 3rd edition, 2007.

[27] J. Pinheiro, D. Bates, S. DebRoy, D. Sarkar, and R Core Team, nlme: Linear and Nonlinear Mixed Effects Models, 2015.

[28] A. G. Dimitrov, A. A. Lazar, and J. D. Victor, "Information theory in neuroscience," Journal of Computational Neuroscience, vol. 30, no. 1, pp. 1-5, 2011. 
[29] O. E. Dick and I. A. Svyatogor, "Potentialities of the wavelet and multifractal techniques to evaluate changes in the functional state of the human brain," Neurocomputing, vol. 82, pp. 207-215, 2012.

[30] O. E. Dick, I. A. Svyatogor, V. A. Ishinova, and A. D. Nozdrachev, "Fractal characteristics of the functional state of the brain in patients with anxiuos phobic disorders," Human Physiology, vol. 38, no. 3, pp. 249-254, 2012.

[31] U. Seneviratne, A. Mohamed, M. Cook, and W. D'Souza, “The utility of ambulatory electroencephalography in routine clinical practice: a critical review," Epilepsy Research, vol. 105, no. 1-2, pp. 1-12, 2013. 


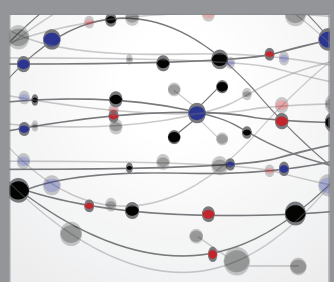

The Scientific World Journal
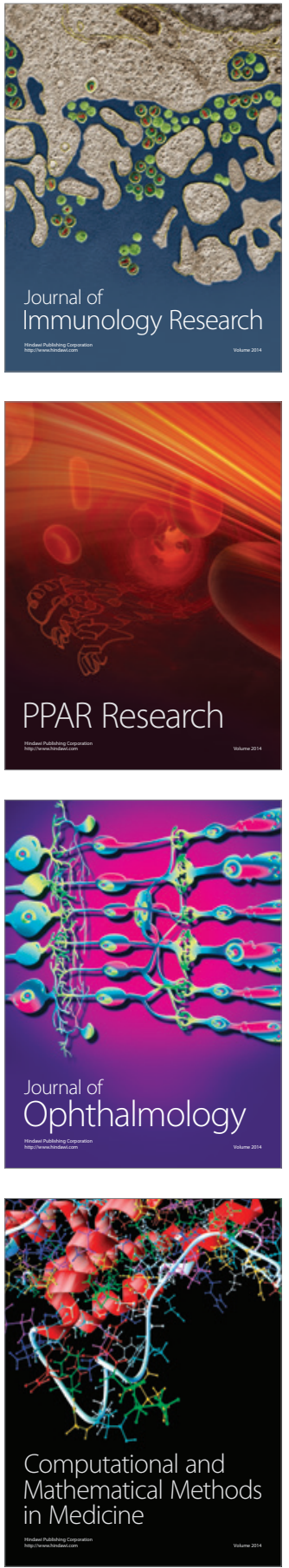

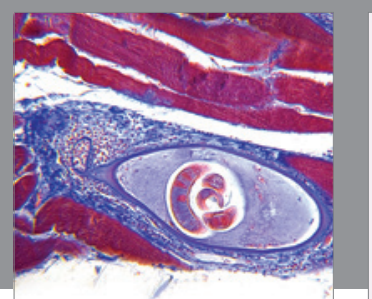

Gastroenterology Research and Practice

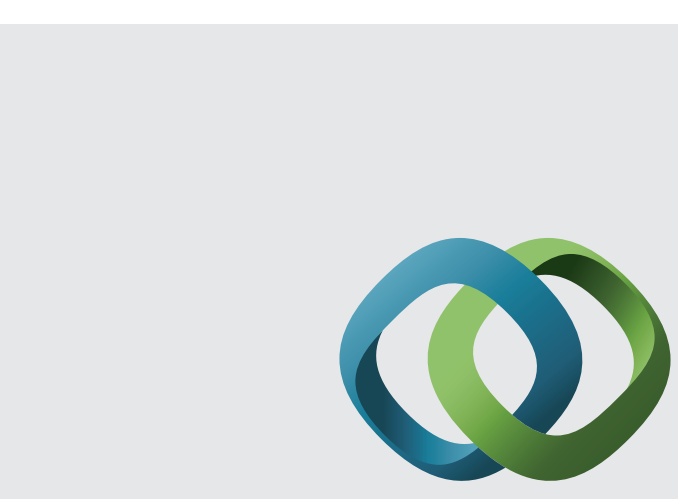

\section{Hindawi}

Submit your manuscripts at

http://www.hindawi.com
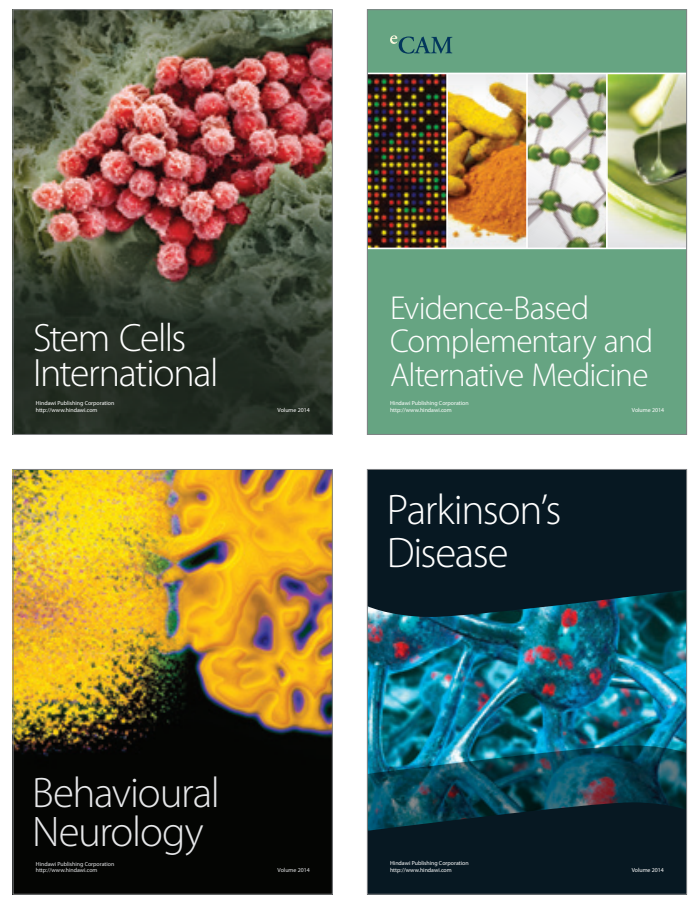
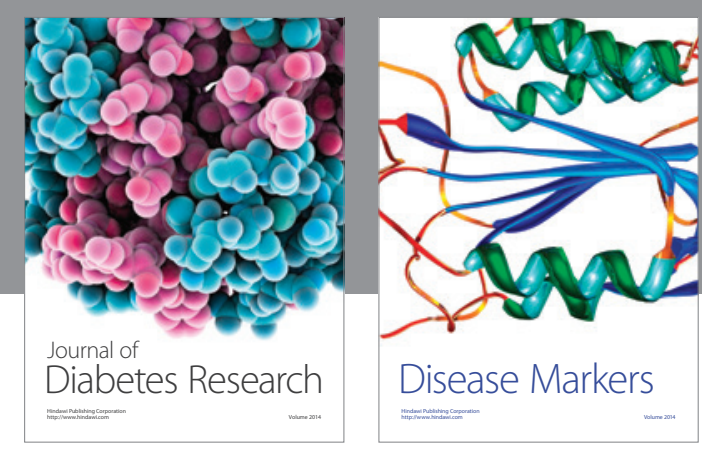

Disease Markers
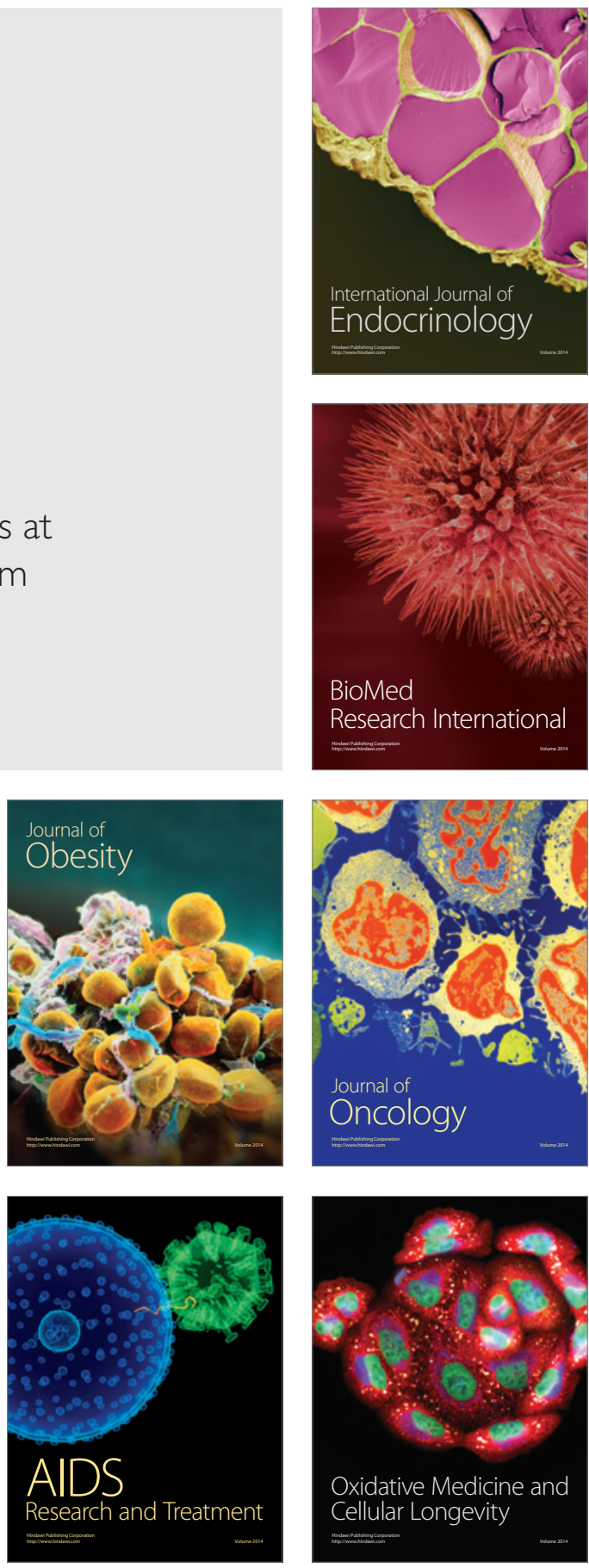